\title{
Variables Antropométricas y Rendimiento Físico en Estudiantes Universitarios de Educación Física
}

\author{
Anthopometric Measures and Physical Performance in University Students of Physical Education
}

*Atilio Aldo Almagià Flores; "Pablo José Lizana Arce; "Fernando Javier Rodríguez Rodríguez;

"Daniza Ivanovic Marincovich \& *** Octavio Binvignat Gutiérrez

ALMAGIA, F. A. A.; LIZANA, A. P. J.; RODRIGUEZ, R. F. J.; IVANOVIC, M. D. \& BINVIGNAT, G. O. Variables antropométricas y rendimiento físico en estudiantes universitarios de educación física. Int. J. Morphol., 27(4):971-975, 2009.

RESUMEN: La estructura corporal del estudiante universitario de Educación Física cobra vital importancia debido a que será relevante en sus logros en las asignaturas que impliquen esfuerzo físico. Consistente con esto, es deseable contar con datos de las características morfoestructurales basadas en el somatotipo y la composición corporal de los estudiantes de ambos sexos que ingresan a estudiar a esta carrera, cotejados con pruebas físicas. Se estudiaron 32 hombres y 18 mujeres, en edades entre 17 y 25 años, de la carrera de Educación Física, de la Pontificia Universidad Católica de Valparaíso, Chile. Se aplicaron los métodos de Composición Corporal de Deborah Kerr y de Somatotipo de Heath-Carter, y se correlacionó con las pruebas físicas de: carrera de 2400 m de George - Fisher, salto vertical con pies juntos, bíceps en tracción vertical, prueba de abdominales y $50 \mathrm{~m}$ de velocidad. Los datos indican que el grupo masculino con un mayor porcentaje de masa grasa realizan la prueba de $50 \mathrm{~m}$ de velocidad en un mayor tiempo $(\mathrm{r}=0,57)$ e inversamente, quienes tienen menor masa grasa, realizaron mayor cantidad de repeticiones en la prueba de barra $(\mathrm{r}=-0,55)$, en el grupo femenino se encuentra una correlación media entre la masa muscular y la prueba de $50 \mathrm{~m}, \mathrm{r}=0,52$. Sin embargo, no existen correlaciones suficientes entre la masa muscular y las pruebas de rendimiento, en las que se esperaba que la cantidad de masa muscular influyera sobre las pruebas que dependen de este componente. Las variables antropométricas del grupo universitario evaluado, no se correlacionan de manera significativa con el rendimiento físico, puesto que el nivel de entrenamiento es bajo, lo que determina el nivel de rendimiento, a diferencia de lo que ocurre con deportistas.

PALABRAS CLAVE: Somatotipo; Composición corporal; Estudiantes; Educación Física.

\section{INTRODUCCIÓN}

El término aptitud física se hizo popular durante la segunda guerra mundial e inicialmente tenía el exclusivo propósito de definir las capacidades físicas de los soldados a través de test físicos. Posteriormente, evolucionaría hasta introducirse en otros ámbitos de la sociedad con la finalidad de aumentar la fuerza muscular, resistencia cardiovascular, pérdida de tejido adiposo, entre otras variables (Monteiro et al., 1994). Consistente con esto, el ejercicio físico incide sobre nuestra estructura corporal, así como nuestra morfoestructura influye sobre nuestro rendimiento. Esta forma física está determinada por el Somatotipo, es decir, la conformación morfológica, forma, tamaño, composición y proporcionalidad del cuerpo (Carter, 1980). En este sentido, la estructura corporal del estudiante universitario de Educación Física cobra importancia debido a que será relevante en el logro de asignaturas que impliquen actividad física.

Estudios en nuestro país indican que los estudiantes varones de primeros años de universidades de la V Región Valparaíso, Chile, resultan con predominancia mesomórfica, pero en menor medida comparados con universidades extranjeras (Toro et al., 1983). Sin embargo, sería deseable contar, con una base de datos de las características morfoestructurales basadas en el somatotipo y la composición corporal de los estudiantes de ambos sexos que ingresan a estudiar a esta carrera, cotejados con la evaluación

\footnotetext{
* Laboratorio de Antropología Física y Anatomía Humana, Instituto de Biología, Facultad de Ciencias, Pontificia Universidad Católica de Valparaíso. Chile. ** Instituto de Nutrición y Tecnología de los Alimentos, Universidad de Chile. Chile.

*** Centro Anatómico, Campus Talca, Universidad Autónoma. Chile; Profesor colaborador Laboratorio de Antropología Física y Anatomía Humana,
} Instituto de Biología, Facultad de Ciencias, Pontificia Universidad Católica de Valparaíso. Chile. 
diagnóstica de condición física, estableciendo evaluaciones periódicas durante su carrera. Al respecto, se espera que los sujetos en estudio con un somatotipo ecto- mesomórfico y una composición corporal con predominio de la masa muscular y bajo porcentaje de masa grasa deban obtener un mejor desempeño físico en las evaluaciones diagnósticas.

\section{SUJETOS Y MÉTODO}

Se estudiaron 50 sujetos aleatoriamente seleccionados de un universo de 80, de ambos sexos, 32 hombres (64\%) y 18 mujeres (36\%) en edades que comprendan entre 17 y 25 años, todos correspondientes a estudiantes de primer año de la carrera de Educación Física de la Pontificia Universidad Católica de Valparaíso, año 2007.

Las variables evaluadas fueron: peso, estatura de pie, estatura sentado, perímetros corporales, diámetros óseos y pliegues cutáneos. Para dichas medidas se usó el kit antropométrico Rosscraft SRL, que incluye antropómetros óseos, huincha métrica y plicómetro. Los métodos utilizados son los de Composición Corporal de Dhebora Kerr (Kerr, 1988) y de Somatotipo de Heath-Carter (Carter; Norton 1996), las mediciones fueron realizadas en el hemicuerpo derecho y bajo el protocolo de marcaje y medición de la International Society for the Avancement in Kineanthropometric (ISAK), en condiciones normales de temperatura, a primera hora de la mañana y después del vaciado urinario.

Las pruebas físicas que se aplicaron fueron: Carrera de 2400 metros de George - Fisher que está diseñada para medir la capacidad aeróbica del sujeto a través del volumen máximo de oxígeno $\left(\mathrm{VO}_{2}\right.$ máx $)$, y su fórmula es: $\mathrm{VO}_{2}$ máxi$\mathrm{mo}=100,5+(8,344 \times \mathrm{S})-(0,1636 \times \mathrm{PC})-(1,438 \times \mathrm{T})-$ $(0,9128 x$ FC), donde S: Sexo (0: mujeres, 1 : hombres); PC: Peso corporal; T: Tiempo en minutos; FC: Frecuencia cardiaca. Otra prueba realizada fue la de Salto Vertical con Pies juntos o Detente Vertical (Det ver) para medir la fuerza explosiva de la musculatura de los miembros inferiores. Una tercera prueba fue la de bíceps en tracción vertical (barra). Una cuarta prueba fue la de abdominales (Abd) donde el sujeto tendrá 60 segundos para realizar la mayor cantidad de repeticiones posibles. La prueba final es la de 50 metros de velocidad.

Los datos se registran en planillas de recolección manual y posteriormente en planillas Excel (Microsoft) de computadora para su análisis básico y determinación de promedio, mediana, valor máximo y valor mínimo para la comparación entre grupos y coeficiente de correlación y $\mathrm{r}^{2}$ para establecer relaciones entre las variables medidas. Esta investigación se rigió bajo las normas del programa biológico internacional, donde las personas fueron informadas y se obtuvo su consentimiento.

\section{RESULTADOS}

En la Tabla I, se aprecian los resultados de las pruebas de condición física para el grupo masculino, donde solo los resultados de salto vertical (Det ver) y 2400 m están dentro de la categoría de aceptable y las pruebas de abdominales (abd), barra (bar) y 50 metros $(50 \mathrm{~m}$ ) están bajo el rendimiento mínimo aceptado para la aprobación de la asignatura a la cual pertenece la muestra de sujetos. En la Tabla II, se aprecian los resultados de las pruebas de condición física para grupo femenino, donde solo los resultados de salto vertical (Det ver) están dentro de la categoría de aceptable y las pruebas de abdominales (abd), $2400 \mathrm{~m}$, barra (bar) y 50 metros (50 m) están bajo el rendimiento mínimo aceptado para la aprobación.

Los valores de masa grasa tanto en varones como en damas se encuentran bajos en comparación otros estudiantes universitarios (Tarnus \& Bourdon, 2006; Martins, 2008), según las ecuaciones de Jackson y Pollock (Jackson \& Pollock, 1985), y considerado como normal según SEEDO 2000 (Aranceta, 2001, 2003). Los valores de masa muscular se encuentran altos de acuerdo a otros estudiantes universitarios (Tarnus).

Obtenidos los resultados de las pruebas en hombres, relacionamos las variables medidas, encontrando valores de correlación media entre la masa adiposa y las pruebas de carrera de 50m (Fig. 1), de resistencia de 2400 m (Fig. 2) y en la prueba de fuerza de barra (Fig. 3) y ninguna correlación entre las pruebas físicas y la cantidad de masa muscular (Tabla III).

Los resultados de las pruebas en mujeres, sólo muestran una relación media negativa entre la masa adiposa y la prueba de barra, y una relación media positiva entre la masa muscular y la prueba de $50 \mathrm{~m}$ de carrera rápida (Tabla IV).

\section{DISCUSIÓN}

La antropometría esta relacionada con la condición física humana, porque un determinado rendimiento físico tiene un perfil antropométrico determinado y viceversa (Monteiro). Nuestros resultados no representan fielmente esta afirmación, a pesar de encontrar relaciones entre las varia- 


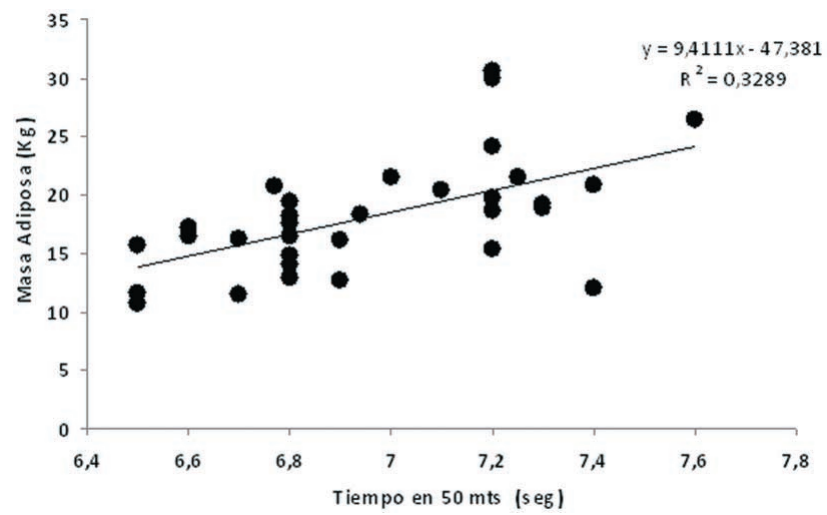

Fig. 1. Gráfica de Pearson y valor $\mathrm{r}^{2}$ entre la masa adiposa y el tiempo en la prueba $50 \mathrm{~m}$ del grupo masculino.

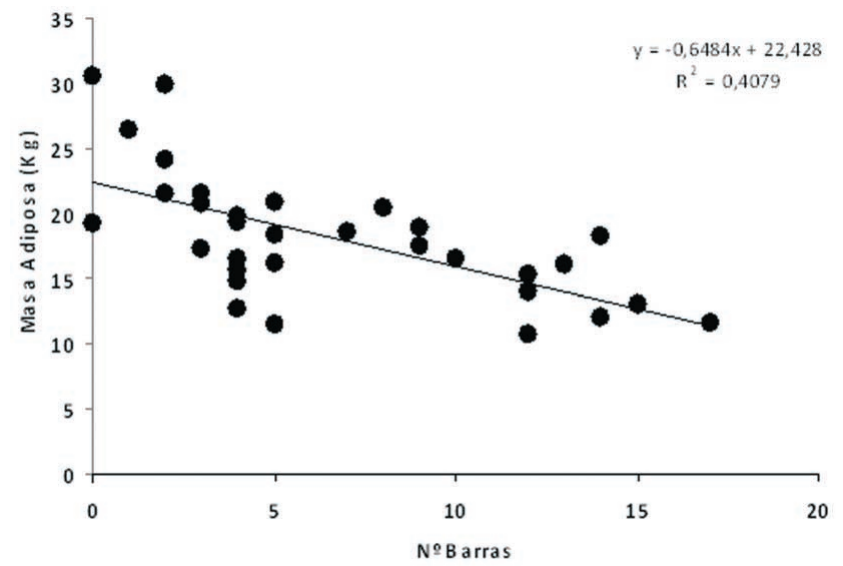

Fig. 3. Gráfica de Pearson y valor $\mathrm{r}^{2}$ entre la masa adiposa y el número de barras realizadas por grupo masculino.

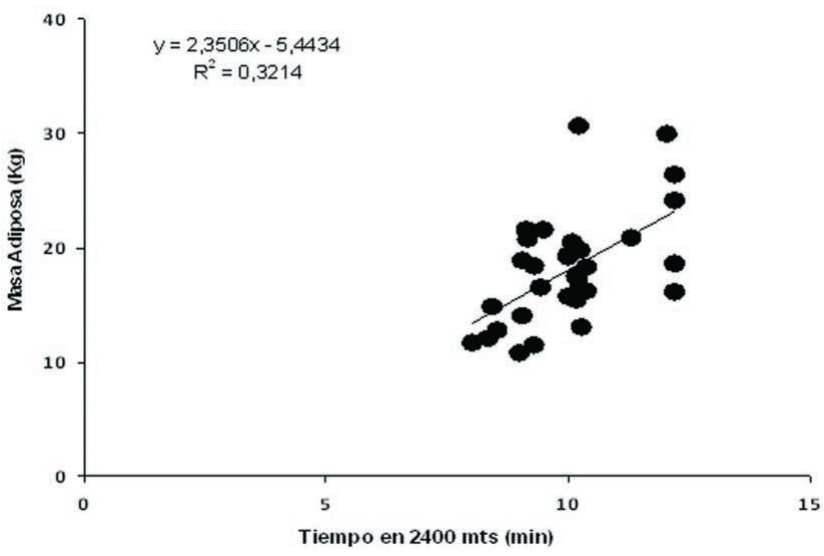

Fig. 2. Gráfica de Pearson y valor $\mathrm{r}^{2}$ entre la masa adiposa y el tiempo en la prueba de $2400 \mathrm{~m}$ del grupo masculino.

bles para el grupo masculino, donde quienes poseen un mayor porcentaje de grasa realizan la prueba de velocidad de $50 \mathrm{~m}$ en un tiempo mayor con un $\mathrm{r}=0,57$. Inversamente, quienes tienen menor cantidad de masa adiposa, fueron capaces de realizar mayor cantidad de repeticiones en la prueba de barra con un $\mathrm{r}=-0,55$ (Tabla III). No hay buenas correlaciones entre la masa muscular y las pruebas de rendimiento, en las cuales se esperaba que la cantidad de masa muscular influyera sobre las pruebas que dependen de este componente, como la carrera de $50 \mathrm{~m}$ o la de salto vertical. Esto contrasta con hallazgos encontrados en jugadores de rugby (Rodríguez et al., 2008) en donde se encon-

Tabla I. Resultados de los test físicos y antropométricos del grupo masculino. MMr \%= porcentaje de masa muscular; MMr Kg=kilogramos de masa muscular; MA \%= porcentaje de masa adiposa; $\mathrm{MA} \mathrm{Kg}=$ kilogramos de masa adiposa; $(\mathrm{seg})=$ tiempo en segundos; $($ min $)=$ minutos; $\left(\mathrm{n}^{\circ}\right)$ número de repeticiones ejecutadas; $(\mathrm{cm})=$ centímetros.

\begin{tabular}{lccccccccc}
\hline & $\begin{array}{c}\text { Det ver } \\
(\mathbf{c m})\end{array}$ & $\begin{array}{c}\text { Abd } \\
\left(\mathbf{n}^{\mathbf{0}}\right)\end{array}$ & $\begin{array}{c}\text { Barra } \\
\left(\mathbf{n}^{\mathbf{0}}\right)\end{array}$ & $\begin{array}{c}\mathbf{2 4 0 0} \mathbf{m} \\
(\mathbf{m i n})\end{array}$ & $\begin{array}{c}\mathbf{5 0} \mathbf{m} \\
(\mathbf{s e g})\end{array}$ & $\mathbf{M M r} \boldsymbol{\%}$ & $\mathbf{M M r ~ K g}$ & MA \% & MA Kg \\
\cline { 2 - 9 } Promedio & $52,4+/-10,5$ & $55,8+/-16,4$ & $6,6+/-5,0$ & $10,0+/-2,0$ & $7,0+/-1,3$ & $49,5+/-9,6$ & $39,1+/-7,9$ & $22,7+/-5,5$ & $18,1+/-5,8$ \\
Mediana & 52,0 & 59,0 & 5,0 & 10,1 & 6,9 & 49,1 & 39,5 & 23,6 & 17,9 \\
Max & 68,0 & 72,0 & 17,0 & 12,2 & 7,6 & 61,8 & 49,0 & 31,0 & 30,6 \\
Min & 40,0 & 20,0 & 0,0 & 8,0 & 6,5 & 41,8 & 27,7 & 15,4 & 10,7 \\
\hline
\end{tabular}

Tabla II. Resultados de los test físicos y antropométricos del grupo femenino.

\section{Det ver Abd Barra $2400 \mathrm{~m} \quad 50 \mathrm{~m}$

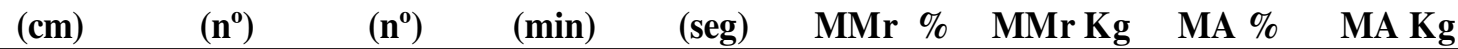

Promedio 37,1+/-9,8 43,9+/-12,5 2,6+/-3,3 $13,2+/-3,2 \quad 8,6+/-2,2 \quad 46,0+/-11,4 \quad 29,0+/-7,9 \quad 28,5+/-7,7 \quad 18,1+/-5,8$

\begin{tabular}{lccccccccc} 
Mediana & 38,5 & 45,5 & 2,0 & 13,4 & 8,4 & 46,1 & 30,7 & 28,8 & 17,6 \\
Max & 48,0 & 60,0 & 11,0 & 14,6 & 10,6 & 55,4 & 35,9 & 41,4 & 24,6 \\
Min & 29,0 & 26,0 & 0,0 & 11,5 & 7,8 & 35,9 & 20,5 & 21,9 & 11,1 \\
\hline
\end{tabular}


ALMAGIA, F. A. A.; LIZANA, A. P. J.; RODRIGUEZ, R. F. J.; IVANOVIC, M. D. \& BINVIGNAT, G. O. Variables antropométricas y rendimiento físico en estudiantes universitarios de educación física. Int. J. Morphol., 27(4):971-975, 2009.

Tabla III. Relación entre los resultados de los test físicos y los resultados antropométricos del grupo masculino. Coeficiente de Correlación.

\begin{tabular}{ccccc}
\hline Pruebas & M. Adiposa $(\mathbf{K g})$ & M. Adiposa (\%) & M. Muscular (kg) & M. Muscular $(\%)$ \\
\hline $\mathbf{5 0 ~} \mathbf{~ m}$ & 0,57 & 0,53 & 0,3 & $-0,12$ \\
$\mathbf{2 4 0 0} \mathbf{~ m}$ & 0,56 & 0,52 & 0,18 & $-0,38$ \\
Barras $\left(\mathbf{n}^{\circ}\right)$ & $-0,55$ & $-0,49$ & $-0,25$ & 0,38 \\
Abd & $-0,38$ & $-0,18$ & $-0,2$ & 0,2 \\
Det ver & $-0,31$ & $-0,29$ & 0,05 & $-0,009$ \\
\hline
\end{tabular}

Tabla IV. Relación entre los resultados de los test físicos y los resultados antropométricos del grupo femenino. Coeficiente de Correlación.

\begin{tabular}{ccccc}
\hline Pruebas & M. Adiposa $(\mathbf{K g})$ & M. Adiposa $(\boldsymbol{\%})$ & M. Muscular $(\mathbf{k g})$ & M. Muscular $(\%)$ \\
\hline $\mathbf{5 0} \mathbf{~ m}$ & 0,2 & 0,26 & $-0,07$ & 0,52 \\
$\mathbf{2 4 0 0} \mathbf{~ m}$ & 0,09 & 0,14 & $-0,11$ & $-0,18$ \\
Barras $\left(\mathbf{n}^{\circ}\right)$ & $-0,34$ & $-0,49$ & 0,16 & 0,46 \\
Abd & $-0,36$ & $-0,18$ & $-0,17$ & 0,22 \\
Det ver & $-0,24$ & $-0,29$ & 0,03 & 0,25 \\
\hline
\end{tabular}

traron buenas correlaciones entre la masa muscular y el salto vertical, $\mathrm{r}=0,72 \mathrm{u}$ otras variables, como por ejemplo, el perímetro del brazo contraído que corresponde una variable para la cuantificación de la masa muscular (Clarys, 1984), con la fuerza máxima extensora del miembro superior, $\mathrm{r}=0,98$.

La explicación a estas diferencias se debe a que la cantidad promedio de los deportistas jugadores de rugby poseen un promedio de $48 \mathrm{~kg}$ de masa muscular a diferencia del promedio de $39 \mathrm{~kg}$ de masa muscular en el grupo de estudiantes, aunque ambos grupos poseían similares porcentajes de este componente, cercano al $50 \%$.

Por lo tanto, se denota que si existe una relación entre los componentes antropométricos y el rendimiento físico, pero que es más marcado en deportistas que en no deportistas, considerando que el grupo de universitarios evaluados solo llevaba dos semanas de entrenamiento físico al momento de ser evaluados, siendo en ese momento sujetos activos, no deportistas.

En el grupo femenino si se encuentra una correlación media entre la masa muscular y la prueba de $50 \mathrm{~m}$, $r=0,52$, la cual depende de la masa muscular. No hay correlaciones entre las otras pruebas físicas y las variables antropométricas, no porque estas últimas sean inadecuadas, sino que debido a que los rendimientos obtenidos fueron muy deficientes, es decir, que el entrenamiento influ- ye sobre el rendimiento físico (Mermier, 2000). Este nivel de entrenamiento modifica la morfoestructura que es cuantificable con la antropometría y que puede utilizarse para determinar y predecir indirectamente el rendimiento físico (Mohamed et al., 2009).

Las variables antropométricas del grupo universitario evaluado, no se correlacionan de manera importante con el rendimiento físico, ya que el nivel de entrenamiento es bajo, el cual determina también el nivel de rendimiento, a diferencia de lo que ocurre con deportistas, quienes si correlacionan variables morfoestructurales con rendimientos físicos. Será necesario aumentar los tipos de poblaciones de estudio para establecer en que punto de condición física comienzan a correlacionarse estos aspectos para aumentar el conocimiento de esta área.

\section{AGRADECIMIENTOS}

Por el apoyo y financiamiento otorgado, a la Dirección de Investigación, Vice-rectoría de Investigación y Estudios Avanzados e Instituto de Biología de la Facultad de Ciencias. Pontificia Universidad Católica de Valparaíso. Chile. A los Profesores de la Escuela de Educación Física, Pontificia Universidad Católica de Valparaíso, Chile, Jaime Silva Estay y Hugo Henríquez Beca por su valioso aporte en la evaluación de pruebas de la condición física. 
ALMAGIA, F. A. A.; LIZANA, A. P. J.; RODRIGUEZ, R. F. J.; IVANOVIC, M. D. \& BINVIGNAT, G. O. Anthopometric measures and physical performance in university students of physical education. Int. J. Morphol., 27(4):971-975, 2009.

SUMMARY: The corporal structure of the university student of Physical education is of vital importance because it will be excellent in it's profits in the subjects that imply physical effort. Consistent with this, is desirable to count on data of the morpho-structural characteristics based on the Stero type and the corporal composition of the students of both sexes that enter to study to this race, collated with physical tests. 32 men and 18 women studied, in ages between 17 and 25 years, of the race of Physical education, Pontificia Universidad Católica de Valparaiso, Chile. They were applied to the methods of Corporal Composition of Dhebora Kerr and somatotype of Heath-Carter, and he correlated himself with the physical tests of: race of $2400 \mathrm{~m}$ of George - Fisher, vertical jump with together feet, biceps in vertical traction, test of abdominal and $50 \mathrm{~m}$ of speed. The data indicate that the masculine group with a greater percentage of greasy mass realises the test of $50 \mathrm{~m}$ of speed in a greater time $(\mathrm{r}=$ $0.57)$ and inversely, that have minor greasy mass, they realised major amount of repetitions in the test of bar $(r=-0,55)$, in the feminine group is an average correlation between the muscular mass and the test of $50 \mathrm{~m}, \mathrm{r}=0,52$. Nevertheless, sufficient correlations between the muscular mass and the performance tests do not exist, in which it hoped that the amount of muscular mass influenced the tests that depend on this component. The anthropometric variables of the evaluated university group, do not correlate of significant way with the physical yield, since the training level is low, which determines the yield level, unlike which it happens with sportsmen.

KEY WORDS: Somatotype; Corporal composition; Students; Physical education.

\section{REFERENCIAS BIBLIOGRÁFICAS}

Aranceta, J.; Pérez, C.; Serra, L.; Ribas, L.; Quiles, J.; Vioque, J.; Tur, J.; Mataix, J.; Llopis, J.; Tojo, R.; Foz, M. \& Grupo colaborativo para el Estudio de la Obesidad en España. Prevalence of obesity in Spain: results of the SEEDO 2000 study. Med. Clin. (Barc), 120(16):608-12, 2003.

Aranceta, J.; Serra, L. L.; Ortega, R.; Entrala, A. \& Gil, A. Las vitaminas en la alimentación de los españoles. Estudio EVE. Madrid, Médica Panamericana, 2001.

Carter, L. The Heath-Carter somatotype method. 2. Ed. San Diego, San Diego State University Syllabus Service, 1980.

Clarys, J. P.; Martin, A. \& Drinkwater, D. Gross tissue weights in the human body by cadaver dissection. Hum. Biol., 56(3):459-73, 1984

Jackson, A. S. \& Pollock, M. L. Practical assessment of body composition. Phys. Sportmed., 13(5):76, 1985.

Kerr, D. A. An anthropometric method for the fractionation of skin, adipose, muscle, bone and residual tissue masses in males and females age 6 to 77 years. M. Sc. Thesis. British Columbia, Simon Fraser University, 1988.

Monteiro, H. L. \& Goncalves, A. Salud colectiva y actividad física: Evolución de las principales concepciones y prácticas. Revista de Ciencias de la Actividad Física, Chile, 3:3345, 1994.

Martins, F.; de Castro, M. H.; de Santana, G. \& Oliveira, L. G. Estado nutricional, medidas antropométricas, nivel socioeconómico y actividad física en universitarios brasileños. Nutr. Hosp., 23(3):234-41, 2008.
Mermier, C. M.; Janot, J. M.; Parker, D. L. \& Swan, J. G. Physiological and anthropometric determinants of sport climbing performance. Br. J. Sports Med., 34(5):359-66, 2000 .

Mohamed, H.; Vaeyens, R.; Matthys, S.; Multael, M.; Lefevre, J.; Lenoir, M. \& Philppaerts, R. Anthropometric and performance measures for the development of a talent detection and identification model in youth handball. J. Sports Sci., 27(3):257-66, 2009.

Norton, K. \& Olds, T. Antropometrica. Marrickville, Sidney, Southwood Press, 1996.

Rodríguez, F.; García, S.; Barraza, F.; Cabrera, C.; Siviero, E. Variables antropométricas y su relación con el rendimiento físico en jugadores de rugby. http://www.efdeportes.com/ Revista Digital - Buenos Aires, 127, 2008.

Tarnus, E. \& Bourdon, E. Anthropometric evaluations of body composition of undergraduate students at the University of La Reunion. Adv. Physiol. Educ., 30(4):248-53, 2006.

Toro, T.; Arenas G. \& Almagia, A. Somatotipo de estudiantes varones de educación física en universidades de Valparaíso. Anal. Anat. Normal, 1:109-10, 1983.

Dirección para correspondencia:

Prof. Atilio Aldo Almagiá Flores

Avda. Brasil 2950 Casilla 4059

Pontificia Universidad Católica de Valparaíso

Valparaíso

CHILE

Email: aalmagia@ucv.cl

Recibido : 29-05-2009

Aceptado: 01-09-2009 
